Homology, Homotopy and Applications, vol.16(1), 2014, pp.199-214

\title{
EXACT SEQUENCES OF COMMUTATIVE MONOIDS AND SEMIMODULES
}

\author{
JAWAD Y. ABUHLAIL \\ (communicated by George Janelidze)
}

\begin{abstract}
Basic homological lemmas well known for modules over rings and, more generally, in the context of abelian categories, have been extended to many other concrete and abstract-categorical contexts by various authors. We propose a new such extension, specifically for commutative monoids and semimodules; these two contexts are equivalent since the forgetful functors from varieties of semimodules to the variety of commutative monoids preserve all limits and colimits.
\end{abstract}

\section{Introduction}

The purpose of this paper is to develop a semimodule version of the collection of basic homological lemmas, including the 5 -Lemma, the $(3 \times 3)$-Lemma, and the Snake Lemma, for commutative monoids and semimodules. In doing so, we use a strong notion of exactness, different from those used by M. Takahashi [18], A. Patchkoria [13], and K.B. Patil and R.P. Deore [15], which are also different from each other. The paper is organized as follows:

- We begin by explaining that both contexts, of commutative monoids and of semimodules, are important in spite of the fact that they are equivalent (Section 1).

- We consider various special classes of morphisms and various notions of an exact sequence of semimodules in Section 2.

- Section 3 is devoted to the above-mentioned homological lemmas.

- Various additional remarks are made in Section 4.

Let us recall here that:

- a semiring $S=(S, 0,+, 1, \cdot)$ is an algebraic structure in which $(S, 0,+)$ and $(S, 1, \cdot)$ are monoids, with commutative + and with $x(y+z)=x y+x z$ (where $x y=x \cdot y$, etc. $),(x+y) z=x z+y z$, and $0 x=0=x 0$ for all $x, y, z \in S$;

The author would like to acknowledge the support provided by the Deanship of Scientific Research (DSR) at King Fahd University of Petroleum \& Minerals (KFUPM) for funding this work through project No. FT100004.

Received March 5, 2013, revised December 3, 2013; published on May 23, 2014.

2010 Mathematics Subject Classification: 18A30, 18G60, 16Y60.

Key words and phrases: exact sequence, semimodule, semiring, commutative monoid, homological lemma, snake lemma.

Article available at http://dx.doi.org/10.4310/HHA.2014.v16.n1.a12

Copyright (C) 2014, International Press. Permission to copy for private use granted. 
- for a semiring $S$, an $S$-semimodule is a commutative monoid $A$ equipped with a map $S \times A \longrightarrow A$, written as $(s, a) \mapsto s a$, and satisfying $s(a+b)=s a+s b$, $(s+t) a=s a+t a,(s \cdot t) a=s(t a), 0 a=0$, and $s 0=0$ for all $s, t \in S$ and $a, b \in$ $A$.

Our main references for semimodules over semirings and categories are $[\mathbf{6}]$ and $[\mathbf{1 2}]$ (or $[\mathbf{1 7}]$ ), respectively.

\section{Why commutative monoids and semimodules?}

1.1. As has been well known for a long time, the following conditions on a variety $\mathbf{A}$ of universal algebras (considered as a category) are equivalent:

(a) $\mathbf{A}$ is enriched in the monoidal closed category of abelian groups; that is, there exist abelian group structures on all hom sets $\operatorname{Hom}_{\mathbf{A}}(A, B)(A, B \in \mathbf{A})$, such that the composition of morphisms distributes over addition on both sides;

(b) $\mathbf{A}$ is an additive category;

(c) $\mathbf{A}$ is an abelian category;

(d) $\mathbf{A}$ is the category of $R$-modules for some ring $R$.

1.2. There is a less known similar result on semimodules. It says that the following conditions on a variety $\mathbf{A}$ of universal algebras are equivalent:

(a) $\mathbf{A}$ is enriched in the monoidal closed category of commutative monoids; that is, there exist commutative monoid structures on all hom sets $\operatorname{Hom}_{\mathbf{A}}(A, B)$ $(A, B \in \mathbf{A})$, such that the composition of morphisms distributes over addition on both sides;

(b) $\mathbf{A}$ is the category of $S$-semimodules for some semiring $S$.

Both of these results are proved (using different terminology) in [5], and, moreover, at least Csákány's proof of 1.2 seems to be the first known such proof.

Observation 1.3. Concerning the so-called basic homological lemmas, we should observe the following. While in the situation 1.1 they have clear unique formulations that belong to classical homological algebra, in the situation 1.2 the formulations might depend on the chosen notion of an exact sequence. However, as soon as the notion of an exact sequence is defined categorically, using limits and colimits (only), each such lemma will hold for semimodules if and only if it holds for commutative monoids. This follows from the fact that the forgetful functor

\section{$U: S$-SMod $\longrightarrow$ CMon}

from the category $S$-SMod of $S$-semimodules to the category CMon of commutative monoids preserves limits and colimits and reflects isomorphisms; in particular, a diagram $A \longrightarrow B \longrightarrow C$ in $S$-SMod is an exact sequence (in the chosen sense) if and only if so is its $U$-image $U(A) \longrightarrow U(B) \longrightarrow U(C)$. Therefore, although all our results apply to the situation 1.2 , it suffices to prove them for commutative monoids, making both these structures fundamentally important for our purposes. 


\section{Four notions of an exact sequence of semimodules}

Considering semimodules over an arbitrary fixed semiring $S$, we keep in mind Observation 1.3, according to which every argument we use reduces to the case of commutative monoids; that is, to the case where $S$ is the semiring of natural numbers. Proposition 2.2 below is well known; it describes several categorically defined classes of morphisms in $S$-SMod in classically algebraic terms. The readers less familiar with category theory can use these descriptions as definitions. Before formulating Proposition 2.2, let us explain our notation and terminology for kernels and cokernels:

2.1. On the one hand, we shall use classical-algebraic notation for kernels and cokernels: for a morphism $f: A \longrightarrow B$ in $S$-SMod, we write

$$
\begin{aligned}
\operatorname{Ker}(f) & =\{a \in A \mid f(a)=0\}, \\
\operatorname{Coker}(f) & =B /\left\{\left(b_{1}, b_{2}\right) \in B \times B \mid\left(\exists a_{1}\right)\left(\exists a_{2}\right)\left(b_{1}+f\left(a_{1}\right)=b_{2}+f\left(a_{2}\right)\right)\right\},
\end{aligned}
$$

and write $\operatorname{ker}(f): \operatorname{Ker}(f) \longrightarrow A$ and $\operatorname{coker}(f): B \longrightarrow \operatorname{Coker}(f)$ for the corresponding canonical maps. On the other hand, we shall use the categorical notation and terminology, according to which $\operatorname{ker}(f): \operatorname{Ker}(f) \longrightarrow A$ and $\operatorname{coker}(f): B \longrightarrow \operatorname{Coker}(f)$ are defined (up to isomorphism) via their universal properties; we will also say that $\operatorname{ker}(f)$ is the kernel of $f$ and $\operatorname{coker}(f)$ is the cokernel of $f$. The classical-algebraic and the categorical-algebraic notations agree up isomorphism, of course.

Proposition 2.2. A morphism $f: A \longrightarrow B$ in $S$-SMod is:

(a) a monomorphism, if and only if $f$ an injective map;

(b) a normal monomorphism, that is, a kernel of some morphism, if and only if ( $f$ is injective and) whenever $b+f\left(a_{1}\right)=f\left(a_{2}\right)$ for $a_{1}, a_{2} \in A$ and $b \in B$, there exists a unique $a \in A$ with $f(a)=b$;

(c) a regular epimorphism if and only if $f$ is a surjective map;

(d) a normal epimorphism, that is, a cokernel of some morphism, if and only if it is surjective and

$$
f\left(a_{1}\right)=f\left(a_{2}\right) \text { if and only if there exist } k_{1}, k_{2} \in \operatorname{Ker}(f) \text { with } a_{1}+k_{1}=a_{2}+k_{2} .
$$

(e) a pullback stable normal epimorphism, whenever it is a normal epimorphism.

For an arbitrary morphism $f: A \longrightarrow B$ in $S$-SMod, we have the canonical factorization

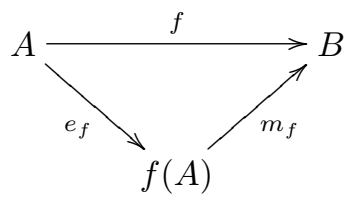

in which $e_{f}: A \longrightarrow f(A)$ is induced by $f$ and $m_{f}: f(A) \longrightarrow B$ is the inclusion map. 
2.3. For morphisms $f: A \longrightarrow B$ and $g: B \longrightarrow C$ in $S$-SMod, consider the diagram

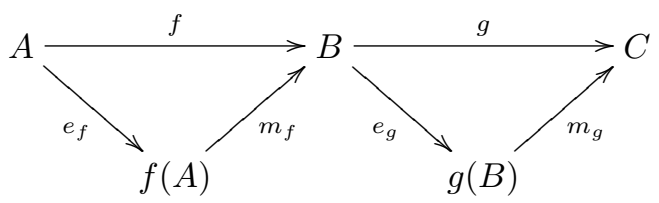

For its top row $A \longrightarrow B \longrightarrow C$ we have

(a) $A \longrightarrow B \longrightarrow C$ is Takahashi exact, that is, exact in the sense of [18], if and only if the kernel of $g$ is the normal closure of $m_{f}$, that is, $\operatorname{Ker}(g)=\operatorname{Ker}(\operatorname{coker}(f))$;

(b) $A \longrightarrow B \longrightarrow C$ is Patchkoria exact, that is, exact in the sense of [13], if and only if $m_{f}$ is the kernel of $g$, that is, $\operatorname{Ker}(g)=f(A)$;

(c) $A \longrightarrow B \longrightarrow C$ is Patil-Deore exact, that is, exact in the sense of [15], if and only if $e_{g}$ is the cokernel of $f$.

This shows the Patchkoria exactness and the Patil-Deore exactness dual to each other and makes both of them more restrictive than Takahashi exactness. We are going, however, to use an even more restrictive notion, namely:

Definition 2.4. In the notation of 2.3 , we will say that $A \stackrel{f}{\longrightarrow} B \stackrel{g}{\longrightarrow} C$ is exact if $m_{f}$ is the kernel of $g$ and $e_{g}$ is the cokernel of $f$, or, equivalently, $A \stackrel{f}{\longrightarrow} B \stackrel{g}{\longrightarrow} C$ is Patchkoria exact and Patil-Deore exact at the same time.

As follows from previous observations, this notion of exactness has the following classical-algebraic reformulation:

Proposition 2.5. In the notation of 2.3, $A \stackrel{f}{\longrightarrow} B \stackrel{g}{\longrightarrow} C$ is exact if and only if $\operatorname{Ker}(g)=f(A)$ and whenever $g\left(b_{1}\right)=g\left(b_{2}\right)$ there exist $a_{1}, a_{2} \in A$ with $b_{1}+f\left(a_{1}\right)=$ $b_{2}+f\left(a_{2}\right)$.

2.6. As usually:

(a) a diagram $\ldots \longrightarrow A \longrightarrow B \longrightarrow C \longrightarrow \ldots$ is said to be exact in $B$ if $A \longrightarrow B \longrightarrow$ $C$ is exact;

(b) a diagram $0 \longrightarrow A \longrightarrow B \longrightarrow C \longrightarrow 0$ is said to be a short exact sequence, if it is exact in $A$, in $B$, and in $C$.

(c) a diagram $A_{0} \longrightarrow A_{1} \longrightarrow \ldots \longrightarrow A_{n-1} \longrightarrow A_{n}$ is an exact sequence, if it is exact in $A_{i}$ for every $i=1, \ldots, n-1$.

Corollary 2.7. In the notation of 2.3, we have:

(a) $0 \longrightarrow A \longrightarrow B$ is exact if and only if the map $A \longrightarrow B$ is injective;

(b) $B \longrightarrow C \longrightarrow 0$ is exact if and only if the map $B \longrightarrow C$ is surjective.

\section{Homological lemmas}

In this section we prove basic homological lemmas for semimodules over an arbitrary fixed semiring $S$. As explained in Section 1, and mentioned again at the beginning of Section 2, we could equivalently do that for commutative monoids, that is, in the special case where $S$ is the semiring of natural numbers. 
Note that according to Proposition 2.2(b), the normal closure $\bar{A}$ of a subsemimodule $A$ of a semimodule $B$ is

$\bar{A}=\operatorname{Ker}(\operatorname{coker}(A \longrightarrow B))=\left\{b \in B \mid\right.$ there exist $a_{1}, a_{2} \in A$ with $\left.\left.b+f\left(a_{1}\right)=f a_{2}\right)\right\}$.

More precisely, we will say that $\bar{A}$ is the normal closure of $A$ in $B$.

Next, we will say that a morphism $\varphi: X \longrightarrow Y$ of semimodules is cancellative if so are all elements of its image, that is, if, for $x$ in $X$ and $y_{1}$ and $y_{2}$ in $Y, x+$ $y_{1}=x+y_{2}$ always implies $y_{1}=y_{2}$. Let us also agree that, in our diagram-chasing arguments below, we shall sometimes use elements of semimodules not mentioning the semimodules they belong to, since it will be clear from the context.

\section{The Five Lemma}

The following result can be easily proved using diagram chasing (compare (b) with [14, Lemma 1.9]).

\section{Lemma 3.1. Let}

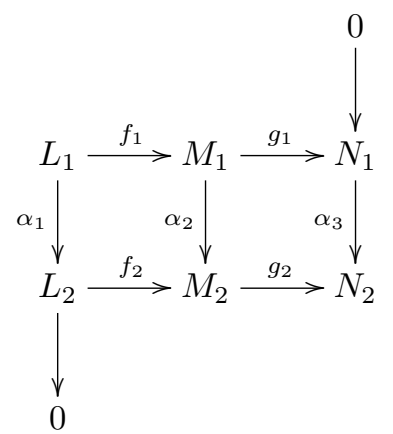

be a commutative diagram of semimodules, in which the first and the third columns are exact. Then

(a) If $\alpha_{2}$ is a regular epimorphism and the first row (formed by $f_{1}$ and $g_{1}$ ) is exact, then the second row (formed by $f_{2}$ and $g_{2}$ ) is exact.

(b) If $\alpha_{2}$ is a monomorphism and the second row is exact, then the first row is exact.

(c) If $a_{2}$ is an isomorphism, then the first row is exact if and only if the second row is exact.

Lemma 3.2. Let

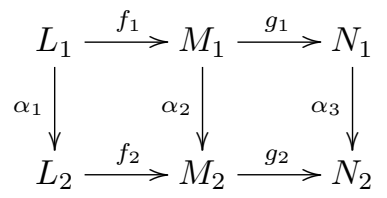

be a commutative diagram of semimodules with exact rows. Then

(a) If $g_{1}$ and $\alpha_{1}$ are regular epimorphisms and $\alpha_{2}$ is a monomorphism, then $\alpha_{3}$ is a monomorphism.

(b) If $f_{2}$ is a monomorphism, $\alpha_{2}$ is a regular epimorphism, and $\alpha_{3}$ has zero kernel, then $\alpha_{1}$ is a regular epimorphism. 
(c) If $f_{2}, \alpha_{1}$, and $\alpha_{3}$ have zero kernels, then $\alpha_{2}$ also has zero kernel.

(d) If $f_{1}$ and $\alpha_{2}$ are cancellative while $f_{2}, \alpha_{1}$ and $\alpha_{3}$ are monomorphisms, then $\alpha_{2}$ also is a monomorphism.

(e) If $g_{1}, \alpha_{1}$, and $\alpha_{3}$ are regular epimorphisms, then the normal closure of $\alpha_{2}\left(M_{1}\right)$ in $M_{2}$ is $M_{2}$ itself.

Proof. (a) Suppose that $\alpha_{3}\left(n_{1}\right)=\alpha_{3}\left(n_{1}^{\prime}\right)$ for some $n_{1}, n_{1}^{\prime} \in N_{1}$. Since $g_{1}$ is a regular epimorphism, $n_{1}=g_{1}\left(m_{1}\right)$ and $n_{1}^{\prime}=g_{1}\left(m_{1}^{\prime}\right)$ for some $m_{1}, m_{1}^{\prime} \in M_{1}$. It follows that $\left(g_{2} \circ \alpha_{2}\right)\left(m_{1}\right)=\left(g_{2} \circ \alpha_{2}\right)\left(m_{1}^{\prime}\right)$. Since the second row is exact, there exist $l_{2}, l_{2}^{\prime} \in L_{2}$ such that $\alpha_{2}\left(m_{1}\right)+f_{2}\left(l_{2}\right)=\alpha_{2}\left(m_{1}^{\prime}\right)+f_{2}\left(l_{2}^{\prime}\right)$. By assumption, $\alpha_{1}$ is a regular epimorphism and so there exist $l_{1}, l_{1}^{\prime} \in L_{1}$ such that $\alpha_{1}\left(l_{1}\right)=l_{2}$ and $\alpha_{1}\left(l_{1}^{\prime}\right)=l_{2}^{\prime}$. Now, we successively obtain the following equalities:

$$
\begin{aligned}
\alpha_{2}\left(m_{1}\right)+\left(f_{2} \circ \alpha_{1}\right)\left(l_{1}\right) & =\alpha_{2}\left(m_{1}^{\prime}\right)+\left(f_{2} \circ \alpha_{1}\right)\left(l_{1}^{\prime}\right), & & \\
\alpha_{2}\left(m_{1}\right)+\left(\alpha_{2} \circ f_{1}\right)\left(l_{1}\right) & =\alpha_{2}\left(m_{1}^{\prime}\right)+\left(\alpha_{2} \circ f_{1}\right)\left(l_{1}^{\prime}\right) & & \\
m_{1}+f_{1}\left(l_{1}\right) & =m_{1}^{\prime}+f_{1}\left(l_{1}^{\prime}\right), & & \left(\alpha_{2} \text { is a monomorphism }\right) \\
g_{1}\left(m_{1}\right) & =g_{1}\left(m_{1}^{\prime}\right), & & \left(g_{1} \circ f_{1}=0\right) \\
n_{1} & =n_{1}^{\prime} . & &
\end{aligned}
$$

(b) Let $l_{2} \in L_{2}$. Since $\alpha_{2}$ is a regular epimorphism, there exists $m_{1} \in M_{1}$ such that $f_{2}\left(l_{2}\right)=\alpha_{2}\left(m_{1}\right)$. It follows that $0=\left(g_{2} \circ f_{2}\right)\left(l_{2}\right)=\left(g_{2} \circ \alpha_{2}\right)\left(m_{1}\right)=\left(\alpha_{3} \circ\right.$ $\left.g_{1}\right)\left(m_{1}\right)$, whence $g_{1}\left(m_{1}\right)=0$ (since $\alpha_{3}$ has zero kernel). Since the first row is exact, $m_{1}=f_{1}\left(l_{1}\right)$ for some $l_{1} \in L_{1}$ and so $f_{2}\left(l_{2}\right)=\alpha_{2}\left(m_{1}\right)=\left(\alpha_{2} \circ f_{1}\right)\left(l_{1}\right)=$ $\left(f_{2} \circ \alpha_{1}\right)\left(l_{1}\right)$. Since $f_{2}$ is a monomorphism, we have $l_{2}=\alpha_{1}\left(l_{1}\right)$.

(c) Suppose that $\alpha_{2}\left(m_{1}\right)=0$ for some $m_{1} \in M_{1}$. We have $\left(\alpha_{3} \circ g_{1}\right)\left(m_{1}\right)=\left(g_{2} \circ\right.$ $\left.\alpha_{2}\right)\left(m_{1}\right)=0$, whence $g_{1}\left(m_{1}\right)=0$ (since $\alpha_{3}$ has zero kernel). Since the first row is exact, $m_{1}=f_{1}\left(l_{1}\right)$ for some $l_{1} \in L_{1}$. So, $0=\alpha_{2}\left(m_{1}\right)=\left(\alpha_{2} \circ f_{1}\right)\left(l_{1}\right)=\left(f_{2} \circ\right.$ $\left.\alpha_{1}\right)\left(l_{1}\right)$, whence $l_{1}=0$ (since $f_{2}$ and $\alpha_{1}$ have zero kernels); consequently, $m_{1}=$ $f_{1}\left(l_{1}\right)=0$.

(d) Suppose that $\alpha_{2}\left(m_{1}\right)=\alpha_{2}\left(m_{1}^{\prime}\right)$ for some $m_{1}, m_{1}^{\prime} \in M_{1}$. We have $\left(\alpha_{3} \circ g_{1}\right)\left(m_{1}\right)=$ $\left(g_{2} \circ \alpha_{2}\right)\left(m_{1}\right)=\left(g_{2} \circ \alpha_{2}\right)\left(m_{1}^{\prime}\right)=\left(\alpha_{3} \circ g_{1}\right)\left(m_{1}^{\prime}\right)$, whence $g_{1}\left(m_{1}\right)=g_{1}\left(m_{1}^{\prime}\right)$ since $\alpha_{3}$ is a monomorphism. Since the first row is exact, there exist $l_{1}, l_{1}^{\prime} \in L_{1}$ such that $m_{1}+f_{1}\left(l_{1}\right)=m_{1}^{\prime}+f_{1}\left(l_{1}^{\prime}\right)$. Now, we successively obtain the following equalities:

$$
\begin{array}{rlrl}
\alpha_{2}\left(m_{1}\right)+\left(\alpha_{2} \circ f_{1}\right)\left(l_{1}\right) & =\alpha_{2}\left(m_{1}^{\prime}\right)+\left(\alpha_{2} \circ f_{1}\right)\left(l_{1}^{\prime}\right), & \\
\alpha_{2}\left(m_{1}^{\prime}\right)+\left(f_{2} \circ \alpha_{1}\right)\left(l_{1}\right) & =\alpha_{2}\left(m_{1}^{\prime}\right)+\left(f_{2} \circ \alpha_{1}\right)\left(l_{1}^{\prime}\right), & & \left(\alpha_{2} \text { is cancellative }\right) \\
\left(f_{2} \circ \alpha_{1}\right)\left(l_{1}\right) & =\left(f_{2} \circ \alpha_{1}\right)\left(l_{1}^{\prime}\right), & & \left(f_{2} \text { and } \alpha_{1} \text { are monomorphisms }\right) \\
l_{1} & =l_{1}^{\prime}, & & \left(f_{1} \text { is cancellative }\right) . \\
m_{1}+f_{1}\left(l_{1}^{\prime}\right) & =m_{1}^{\prime}+f_{1}\left(l_{1}^{\prime}\right), & &
\end{array}
$$

(e) Let $m_{2} \in M_{2}$. Since $g_{1}$ and $\alpha_{3}$ are regular epimorphisms, there exists $m_{1} \in M_{1}$ such that $g_{2}\left(m_{2}\right)=\left(\alpha_{3} \circ g_{1}\right)\left(m_{1}\right)=\left(g_{2} \circ \alpha_{2}\right)\left(m_{1}\right)$. Since the second row is exact and $\alpha_{1}$ is a regular epimorphism, there exist $l_{1}, l_{1}^{\prime} \in L_{1}$ such that

$$
\begin{aligned}
m_{2}+\left(f_{2} \circ \alpha_{1}\right)\left(l_{1}\right) & =\alpha_{2}\left(m_{1}\right)+\left(f_{2} \circ \alpha_{1}\right)\left(l_{1}^{\prime}\right), \\
m_{2}+\alpha_{2}\left(f_{1}\left(l_{1}\right)\right) & =\alpha_{2}\left(m_{1}+f_{1}\left(l_{1}^{\prime}\right)\right),
\end{aligned}
$$

that is, $m_{2} \in \overline{\alpha_{2}\left(M_{1}\right)}$. 
Proposition 3.3 (The Short Five Lemma). Let

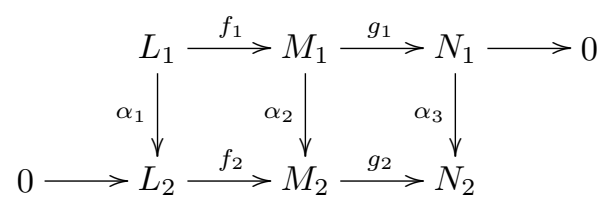

be a commutative diagram of semimodules with exact rows, and assume that $M_{1}$ and $M_{2}$ are cancellative. If $\alpha_{2}\left(M_{1}\right)=\overline{\alpha_{2}\left(M_{1}\right)}$ while $\alpha_{1}$ and $\alpha_{3}$ are isomorphisms, then $\alpha_{2}$ also is an isomorphism.

Corollary 3.4. Let

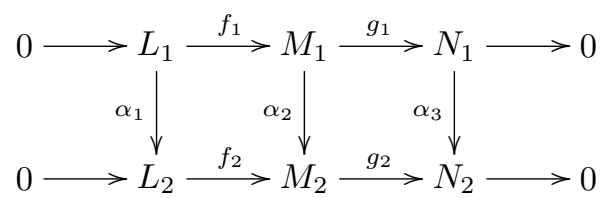

be a commutative diagram of semimodules with exact rows, and assume that $M_{1}$ and $M_{2}$ are cancellative. If $\alpha_{2}\left(M_{1}\right)=\overline{\alpha_{2}\left(M_{1}\right)}$ and any two of $\alpha_{1}, \alpha_{2}$, and $\alpha_{3}$ are isomorphisms, then the third also is an isomorphism.

Lemma 3.5. Let

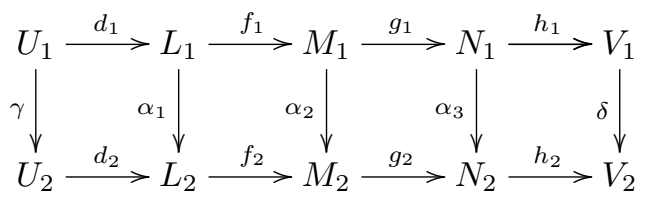

be a commutative diagram of semimodules with exact rows. Then:

(a) If $\gamma$ is a regular epimorphism, $\alpha_{1}$ is a monomorphism and $\alpha_{3}$ has zero kernel, then $\alpha_{2}$ also has zero kernel.

(b) If $\gamma$ is a regular epimorphism, $f_{1}$ and $\alpha_{2}$ are cancellative while $\alpha_{1}$ and $\alpha_{3}$ are monomorphisms, then $\alpha_{2}$ also is a monomorphism.

(c) If $\delta$ has zero kernel while $\alpha_{1}$ and $\alpha_{3}$ are regular epimorphisms, then the normal closure of $\alpha_{2}\left(M_{1}\right)$ in $M_{2}$ is $M_{2}$ itself.

(d) If $\gamma$ is a regular epimorphism, $\delta$ is a monomorphism, $f_{1}$ and $\alpha_{2}$ are cancellative while $\alpha_{1}$ and $\alpha_{3}$ are isomorphisms, then $\alpha_{2}$ is a monomorphism and the normal closure of $\alpha_{2}\left(M_{1}\right)$ is $M_{2}$ is $M_{2}$ itself.

Proof. (a) Suppose that $\alpha_{2}\left(m_{1}\right)=0$ for some $m_{1} \in M_{1}$. We have $\left(\alpha_{3} \circ g_{1}\right)\left(m_{1}\right)=$ $\left(g_{2} \circ \alpha_{2}\right)\left(m_{1}\right)=0$. Since $\alpha_{3}$ has zero kernel, $g_{1}\left(m_{1}\right)=0$ and so $m_{1}=f_{1}\left(l_{1}\right)$ for some $l_{1} \in L_{1}$. It follows that $0=\alpha_{2}\left(m_{1}\right)=\left(\alpha_{2} \circ f_{1}\right)\left(l_{1}\right)=\left(f_{2} \circ \alpha_{1}\right)\left(l_{1}\right)$, whence $\alpha_{1}\left(l_{1}\right)=\left(d_{2} \circ \gamma\right)\left(u_{1}\right)=\left(\alpha_{1} \circ d_{1}\right)\left(u_{1}\right)$ for some $u_{1} \in U_{1}$ (since $\gamma$ is a regular epimorphism and $\left.\operatorname{Ker}\left(f_{2}\right)=d_{2}\left(U_{2}\right)\right)$. Since $\alpha_{1}$ is a monomorphism, $l_{1}=d_{1}\left(u_{1}\right)$, whence $m_{1}=f_{1}\left(l_{1}\right)=\left(f_{1} \circ d_{1}\right)\left(u_{1}\right)=0$.

(b) Suppose that $\alpha_{2}\left(m_{1}\right)=\alpha_{2}\left(m_{1}^{\prime}\right)$ for some $m_{1}, m_{1}^{\prime} \in M_{1}$. We have $\left(\alpha_{3} \circ g_{1}\right)\left(m_{1}\right)=$ $\left(g_{2} \circ \alpha_{2}\right)\left(m_{1}\right)=\left(g_{2} \circ \alpha_{2}\right)\left(m_{1}^{\prime}\right)=\left(\alpha_{3} \circ g_{1}\right)\left(m_{1}^{\prime}\right)$, whence $g_{1}\left(m_{1}\right)=g_{1}\left(m_{1}^{\prime}\right)$ because 
$\alpha_{3}$ is a monomorphism. Since $L_{1} \stackrel{f_{1}}{\longrightarrow} M_{1} \stackrel{g_{1}}{\longrightarrow} N_{1}$ is exact, there exist $l_{1}, l_{1}^{\prime} \in L_{1}$ such that $m_{1}+f_{1}\left(l_{1}\right)=m_{1}^{\prime}+f_{1}\left(l_{1}^{\prime}\right)$. Now, we successively obtain the following equalities:

$$
\begin{array}{rlrl}
\alpha_{2}\left(m_{1}\right)+\left(\alpha_{2} \circ f_{1}\right)\left(l_{1}\right) & =\alpha_{2}\left(m_{1}^{\prime}\right)+\left(\alpha_{2} \circ f_{1}\right)\left(l_{1}^{\prime}\right), & \\
\alpha_{2}\left(m_{1}^{\prime}\right)+\left(f_{2} \circ \alpha_{1}\right)\left(l_{1}\right) & =\alpha_{2}\left(m_{1}^{\prime}\right)+\left(f_{2} \circ \alpha_{1}\right)\left(l_{1}^{\prime}\right), & & \\
f_{2}\left(\alpha_{1}\left(l_{1}\right)\right) & =f_{2}\left(\alpha_{1}\left(l_{1}^{\prime}\right)\right), & & \left(\alpha_{2} \text { is cancellative }\right) \\
\alpha_{1}\left(l_{1}\right)+k_{2} & =\alpha_{1}\left(l_{1}^{\prime}\right)+k_{2}^{\prime}, & & \left(U_{2} \stackrel{d_{2}}{\longrightarrow} L_{2} \stackrel{f_{2}}{\longrightarrow} M_{2} \text { is exact }\right) \\
\alpha_{1}\left(l_{1}\right)+\left(d_{2} \circ \gamma\right)\left(u_{1}\right) & =\alpha_{1}\left(l_{1}^{\prime}\right)+\left(d_{2} \circ \gamma\right)\left(u_{1}^{\prime}\right), & & (\gamma \text { is a regular epimorphism }) \\
\alpha_{1}\left(l_{1}\right)+\left(\alpha_{1} \circ d_{1}\right)\left(u_{1}\right) & =\alpha_{1}\left(l_{1}^{\prime}\right)+\left(\alpha_{1} \circ d_{1}\right)\left(u_{1}^{\prime}\right), & & \\
l_{1}+d_{1}\left(u_{1}\right) & =l_{1}^{\prime}+d_{1}\left(u_{1}^{\prime}\right), & & \left(\alpha_{1} \text { is a monomorphism }\right) \\
f_{1}\left(l_{1}\right) & =f_{1}\left(l_{1}^{\prime}\right),\left(f_{1} \circ d_{1}=0\right) & & \\
m_{1}+f_{1}\left(l_{1}\right) & =m_{1}+f_{1}\left(l_{1}^{\prime}\right), & & \\
m_{1}^{\prime}+f_{1}\left(l_{1}^{\prime}\right) & =m_{1}+f_{1}\left(l_{1}^{\prime}\right), & & \\
m_{1}^{\prime} & =m_{1} . & &
\end{array}
$$

(c) Let $m_{2} \in M_{2}$. Since $\alpha_{3}$ is a regular epimorphism, there exists $n_{1} \in N_{1}$ such that $g_{2}\left(m_{2}\right)=\alpha_{3}\left(n_{1}\right)$. It follows that $0=\left(h_{2} \circ g_{2}\right)\left(m_{2}\right)=\left(h_{2} \circ \alpha_{3}\right)\left(n_{1}\right)=(\delta \circ$ $\left.h_{1}\right)\left(n_{1}\right)$, whence $h_{1}\left(n_{1}\right)=0$ (since $\delta$ has zero kernel). Since $g_{1}\left(M_{1}\right)=\operatorname{Ker}\left(h_{1}\right)$, we have $n_{1}=g_{1}\left(m_{1}\right)$ for some $m_{1} \in M_{1}$. Notice that $\left(g_{2} \circ \alpha_{2}\right)\left(m_{1}\right)=\left(\alpha_{3} \circ\right.$ $\left.g_{1}\right)\left(m_{1}\right)=\alpha_{3}\left(n_{1}\right)=g_{2}\left(m_{2}\right)$. Since $L_{2} \stackrel{f_{2}}{\longrightarrow} M_{2} \stackrel{g_{2}}{\longrightarrow} N_{2}$ is exact and $\alpha_{1}$ is a regular epimorphism, there exists $l_{1}, l_{1}^{\prime} \in L_{1}$ such that

$$
\begin{aligned}
\alpha_{2}\left(m_{1}\right)+\left(f_{2} \circ \alpha_{1}\right)\left(l_{1}\right) & =m_{2}+\left(f_{2} \circ \alpha_{1}\right)\left(l_{1}^{\prime}\right), \\
\alpha_{2}\left(m_{1}+f_{1}\left(l_{1}\right)\right) & =m_{2}+\alpha_{2}\left(f_{1}\left(l_{1}^{\prime}\right)\right),
\end{aligned}
$$

that is, $m_{2} \in \overline{\alpha_{2}\left(M_{1}\right)}$.

(d) This is a combination of (a), (b) and (c).

Proposition 3.6 (The Five Lemma). Let

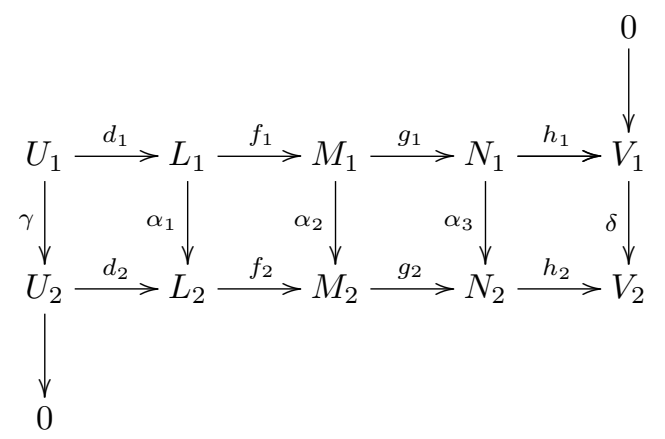

be a commutative diagram of semimodules with exact rows and exact first and fifth columns, and assume that $M_{1}$ and $M_{2}$ are cancellative. Then:

(a) If $\alpha_{1}$ and $\alpha_{3}$ are monomorphisms, then $\alpha_{2}$ also is a monomorphism.

(b) If $\alpha_{2}\left(M_{1}\right)=\overline{\alpha_{2}\left(M_{1}\right)}$ while $\alpha_{1}$ and $\alpha_{3}$ are regular epimorphisms, then $\alpha_{2}$ also is a regular epimorphism.

(c) If $\alpha_{2}\left(M_{1}\right)=\overline{\alpha_{2}\left(M_{1}\right)}$ while $\alpha_{1}$ and $\alpha_{3}$ are isomorphisms, then $\alpha_{2}$ also is an isomorphism. 


\section{The Nine Lemma}

Lemma 3.7. Let

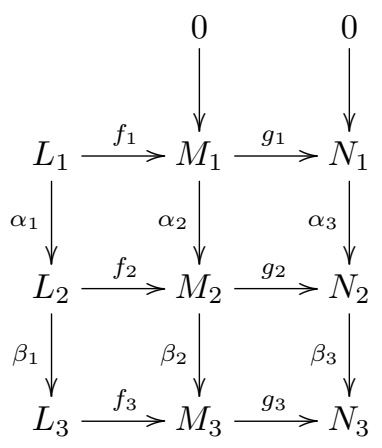

be a commutative diagram with exact columns and exact second row (formed by $f_{2}$ and $\left.g_{2}\right)$. Then:

(a) If $f_{2}$ is cancellative and $f_{3}$ is a monomorphism, then the first row is exact.

(b) If $g_{2}$ and $\beta_{1}$ are regular epimorphisms and the third row is exact, then the normal closure of $g_{1}\left(M_{1}\right)$ in $N_{1}$ is $N_{1}$ itself.

Proof. (a) Notice that $\alpha_{3} \circ g_{1} \circ f_{1}=g_{2} \circ \alpha_{2} \circ f_{1}=g_{2} \circ f_{2} \circ \alpha_{1}=0$, whence $g_{1} \circ f_{1}$ $=0$ since $\alpha_{3}$ is a monomorphism. In particular, $f_{1}\left(L_{1}\right) \subseteq \operatorname{Ker}\left(g_{1}\right)$.

- Suppose that $g_{1}\left(m_{1}\right)=0$. We successively obtain the following equalities:

$$
\begin{aligned}
\left(\alpha_{3} \circ g_{1}\right)\left(m_{1}\right) & =0 & & \\
\left(g_{2} \circ \alpha_{2}\right)\left(m_{1}\right) & =0 & & \\
\alpha_{2}\left(m_{1}\right) & =f_{2}\left(l_{2}\right), & & \\
0 & =\left(\beta_{2} \circ f_{2}\right)\left(l_{2}\right), & & \left(\beta_{2} \circ \alpha_{2}=0\right) \\
0 & =\left(f_{3} \circ \beta_{1}\right)\left(l_{2}\right), & & \\
\beta_{1}\left(l_{2}\right) & =0 & & \\
l_{2} & =\alpha_{1}\left(l_{1}\right), & & \left(\alpha_{1}\left(f_{1}\right)=0\right) \\
f_{2}\left(l_{2}\right) & =\left(f_{2} \circ \alpha_{1}\right)\left(l_{1}\right), & & \\
\alpha_{2}\left(m_{1}\right) & =\alpha_{2}\left(f_{1}\left(l_{1}\right)\right), & & \\
m_{1} & =f_{1}\left(l_{1}\right) & & \left(\alpha_{2} \text { is a monomorphism }\right)
\end{aligned}
$$

- Suppose that $g_{1}\left(m_{1}\right)=g_{1}\left(m_{1}^{\prime}\right)$ for some $m_{1}, m_{1}^{\prime} \in M_{1}$. We then successively obtain the following equalities:

$$
\begin{aligned}
\left(\alpha_{3} \circ g_{1}\right)\left(m_{1}\right) & =\left(\alpha_{3} \circ g_{1}\right)\left(m_{1}^{\prime}\right), & & \\
\left(g_{2} \circ \alpha_{2}\right)\left(m_{1}\right) & =\left(g_{2} \circ \alpha_{2}\right)\left(m_{1}^{\prime}\right), & & \\
\alpha_{2}\left(m_{1}\right)+f_{2}\left(l_{2}\right) & =\alpha_{2}\left(m_{1}^{\prime}\right)+f_{2}\left(l_{2}^{\prime}\right) & & (2 \text { nd row is exact }) \\
\left(\beta_{2} \circ f_{2}\right)\left(l_{2}\right) & =\left(\beta_{2} \circ f_{2}\right)\left(l_{2}^{\prime}\right) & & \left(\beta_{2} \circ \alpha_{2}=0\right) \\
\left(f_{3} \circ \beta_{1}\right)\left(l_{2}\right) & =\left(f_{3} \circ \beta_{1}\right)\left(l_{2}^{\prime}\right), & & \\
\beta_{1}\left(l_{2}\right) & =\beta_{1}\left(l_{2}^{\prime}\right) & & \left(f_{3} \text { is a monomorphism }\right)
\end{aligned}
$$




$$
\begin{aligned}
l_{2}+\alpha_{1}\left(l_{1}\right) & =l_{2}^{\prime}+\alpha_{1}\left(l_{1}^{\prime}\right) \quad(\text { first column is exact }) \\
f_{2}\left(l_{2}\right)+\left(f_{2} \circ \alpha_{1}\right)\left(l_{1}\right) & =f_{2}\left(l_{2}^{\prime}\right)+\left(f_{2} \circ \alpha_{1}\right)\left(l_{1}^{\prime}\right), \\
f_{2}\left(l_{2}\right)+\left(\alpha_{2} \circ f_{1}\right)\left(l_{1}\right) & =f_{2}\left(l_{2}^{\prime}\right)+\left(\alpha_{2} \circ f_{1}\right)\left(l_{1}^{\prime}\right), \\
\alpha_{2}\left(m_{1}\right)+f_{2}\left(l_{2}\right)+\left(\alpha_{2} \circ f_{1}\right)\left(l_{1}\right) & =\alpha_{2}\left(m_{1}\right)+f_{2}\left(l_{2}^{\prime}\right)+\left(\alpha_{2} \circ f_{1}\right)\left(l_{1}^{\prime}\right) \\
f_{2}\left(l_{2}^{\prime}\right)+\alpha_{2}\left(m_{1}^{\prime}+f_{1}\left(l_{1}\right)\right) & =f_{2}\left(l_{2}^{\prime}\right)+\alpha_{2}\left(m_{1}+f_{1}\left(l_{1}^{\prime}\right)\right) \\
\alpha_{2}\left(m_{1}^{\prime}+f_{1}\left(l_{1}\right)\right) & =\alpha_{2}\left(m_{1}+f_{1}\left(l_{1}^{\prime}\right)\right)\left(f_{2} \text { is cancellative }\right) \\
m_{1}^{\prime}+f_{1}\left(l_{1}\right) & =m_{1}+f_{1}\left(l_{1}^{\prime}\right) \quad\left(\alpha_{2} \text { is a monomorphism }\right)
\end{aligned}
$$

The result follows from the fact that $f_{1}\left(L_{1}\right) \subseteq \operatorname{Ker}\left(g_{1}\right)$.

(b) Let $n_{1} \in N_{1}$, and pick $m_{2} \in M_{2}$ such that $g_{2}\left(m_{2}\right)=\alpha_{3}\left(n_{1}\right)$ (by assumption $g_{2}$ is a regular epimorphism). We successively obtain the following equalities:

$$
\begin{aligned}
g_{3}\left(\beta_{2}\left(m_{2}\right)\right) & =\beta_{3}\left(g_{2}\left(m_{2}\right)\right), & & \\
& =\left(\beta_{3} \circ \alpha_{3}\right)\left(m_{2}\right), & & \\
& =0, & & \left(\beta_{3} \circ \alpha_{3}=0\right) \\
\beta_{2}\left(m_{2}\right) & =f_{3}\left(l_{3}\right), & & \left(f_{3}\left(L_{3}\right)=\operatorname{Ker}\left(g_{3}\right)\right) \\
& =f_{3}\left(\beta_{1}\left(l_{2}\right)\right), & & \left(\beta_{1}\right. \text { is a regular epimor } \\
& =\beta_{2}\left(f_{2}\left(l_{2}\right)\right), & & \\
m_{2}+\alpha_{2}\left(m_{1}\right) & =f_{2}\left(l_{2}\right)+\alpha_{2}\left(m_{1}^{\prime}\right), & & (2 \text { nd column is exact }) \\
g_{2}\left(m_{2}\right)+\left(g_{2} \circ \alpha_{2}\right)\left(m_{1}\right) & =\left(g_{2} \circ \alpha_{2}\right)\left(m_{1}^{\prime}\right), & & \left(g_{2} \circ f_{2}=0\right) \\
\alpha_{3}\left(n_{1}+g_{1}\left(m_{1}\right)\right) & =\alpha_{3}\left(g_{1}\left(m_{1}^{\prime}\right)\right), & & \\
n_{1}+g_{1}\left(m_{1}\right) & =g_{1}\left(m_{1}^{\prime}\right), & & \left(\alpha_{3} \text { is injective }\right)
\end{aligned}
$$

that is, $n_{1} \in \overline{g_{1}\left(M_{1}\right)}$.

Similarly, one can prove the following result:

Lemma 3.8. Let

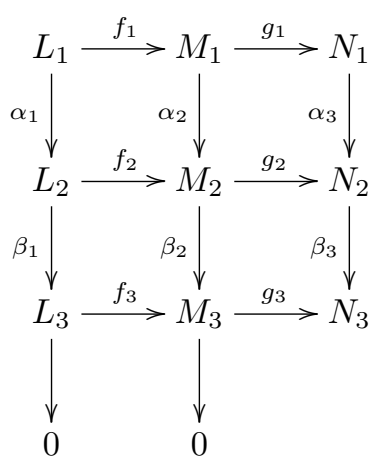

be a commutative diagram with exact columns and exact second row. Then:

(a) If $g_{1}$ is a regular epimorphism and $f_{3}\left(L_{3}\right)=\overline{f_{3}\left(L_{3}\right)}$, then the third row is exact.

(b) If the first row is exact, $\alpha_{2}$ is cancellative while $f_{2}$ and $\alpha_{3}$ are monomorphisms, then $f_{3}$ also is a monomorphism.

The following result is obtained immediately by combining Lemmas 3.7 and 3.8: 
Proposition 3.9 (The Nine Lemma). Let

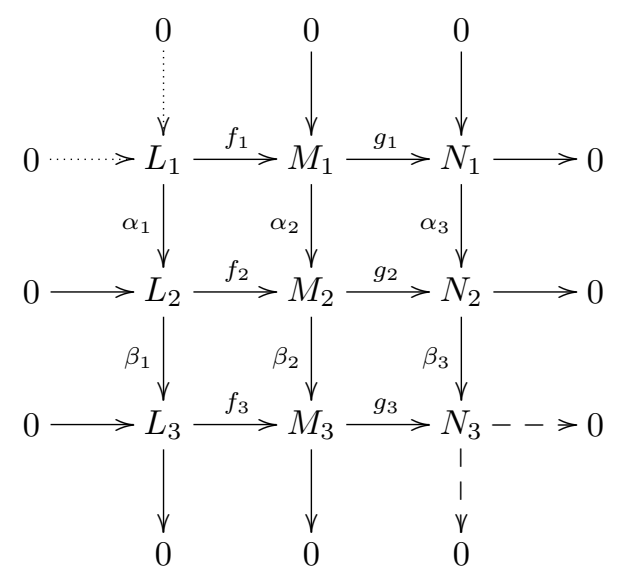

be a commutative diagram with exact columns and exact second row where $M_{2}$ is cancellative. If $f_{3}\left(L_{3}\right)=\overline{f_{3}\left(L_{3}\right)}$ and $g_{1}\left(M_{1}\right)=\overline{g_{1}\left(M_{1}\right)}$, then the first row is exact if and only if the third row is exact.

\section{The Snake Lemma}

One of the basic homological lemmas that are proved usually in categories of modules, or more generally in abelian categories, is the so-called Kernel-Cokernel Lemma (Snake Lemma). Several versions of this lemma were proved also in nonabelian categories that do not include the category of commutative monoids (e.g., homological categories [4], relative homological categories [9], and incomplete relative homological categories $[\mathbf{1 0}])$.

Theorem 3.10 (The Snake Lemma). Let

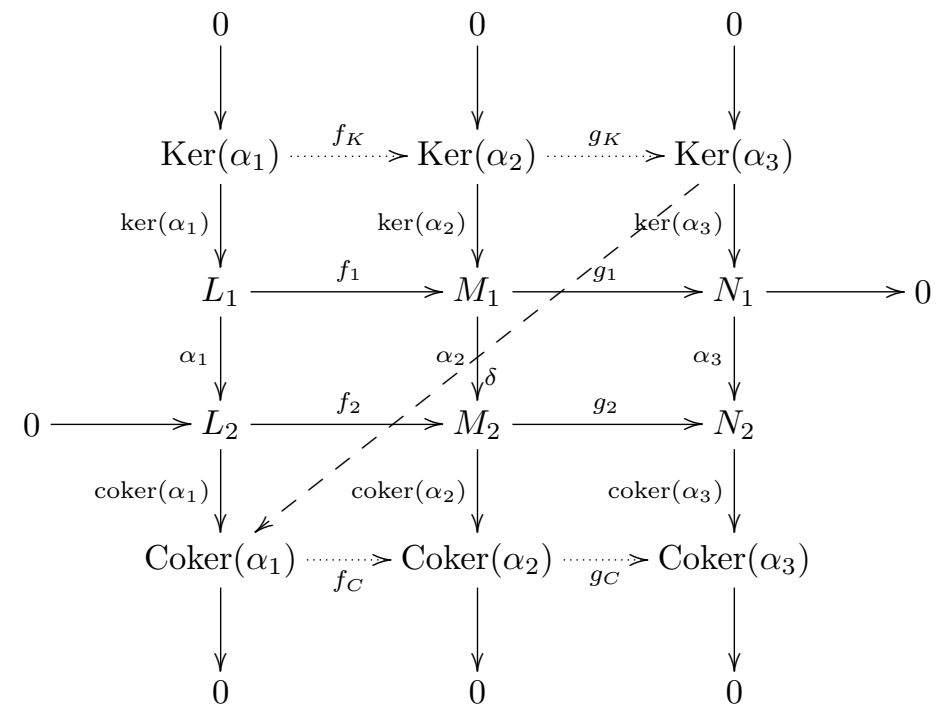

be a diagram of semimodules with exact columns and exact two middle rows, and 
assume that the two middle squares are commutative. Then

(a) There exist unique morphisms $f_{K}, g_{K}, f_{C}$, and $g_{C}$ which extend the diagram commutatively.

(b) If $f_{1}$ is cancellative, then the first row is exact.

(c) If $f_{C}\left(\operatorname{Coker}\left(\alpha_{1}\right)\right)=\overline{f_{C}\left(\operatorname{Coker}\left(\alpha_{1}\right)\right)}$, then the last row is exact.

(d) There exists a connecting morphism $\delta: \operatorname{Ker}\left(\alpha_{3}\right) \longrightarrow \operatorname{Coker}\left(\alpha_{1}\right)$ with $\operatorname{Ker}(\delta)=$ $\overline{g_{K}\left(\operatorname{Ker}\left(\alpha_{2}\right)\right)}, \delta\left(\operatorname{Ker}\left(\alpha_{3}\right)\right)=\operatorname{Ker}\left(f_{C}\right)$, and assume that $\delta\left(k_{3}\right)=\delta\left(k_{3}^{\prime}\right)$ for any $k_{3}$ and $k_{3}^{\prime} \in \operatorname{Ker}\left(\alpha_{3}\right)$ implies that $k_{3}+\widetilde{k}_{3}=k_{3}^{\prime}+\widehat{k}_{3}$ for some $\widetilde{k}_{3}, \widehat{k}_{3} \in \operatorname{Ker}(\delta)$.

(e) If $\alpha_{2}$ is cancellative, and $g_{K}\left(\operatorname{Ker}\left(\alpha_{2}\right)\right)=\overline{g_{K}\left(\operatorname{Ker}\left(\alpha_{2}\right)\right)}$, then the following sequence is exact:

$$
\operatorname{Ker}\left(\alpha_{2}\right) \stackrel{g_{K}}{>}>\operatorname{Ker}\left(\alpha_{3}\right)-\stackrel{\delta}{-}>\operatorname{Coker}\left(\alpha_{1}\right) \stackrel{f_{C}}{>} \operatorname{Coker}\left(\alpha_{2}\right)
$$

Proof. (a) The existence and uniqueness of the morphisms $f_{K}, g_{K}, f_{C}$, and $g_{C}$ are guaranteed by the universal properties of the (co)kernels and the commutativity of the middle two squares.

(b) This follows from Lemma 3.7 applied to the first three rows.

(c) This follows from Lemma 3.8 applied to the last three rows.

(d) We show first that $\delta$ exists and is well defined.

- We define $\delta$ as follows: For $k_{3} \in \operatorname{Ker}\left(\alpha_{3}\right)$, we choose $m_{1} \in M_{1}$ and $l_{2} \in L_{2}$ such that $g_{1}\left(m_{1}\right)=k_{3}$ and $f_{2}\left(l_{2}\right)=\alpha_{2}\left(m_{1}\right)$; notice that this is possible since $g_{1}$ is a regular epimorphism and $\left(g_{2} \circ \alpha_{2}\right)\left(m_{1}\right)=\left(\alpha_{3} \circ g_{1}\right)\left(m_{1}\right)=\alpha_{3}\left(k_{3}\right)=$ 0 whence $\alpha_{2}\left(m_{1}\right) \in \operatorname{Ker}\left(g_{2}\right)=f_{2}\left(L_{2}\right)$. Define $\delta\left(k_{3}\right):=\operatorname{coker}\left(\alpha_{1}\right)\left(l_{2}\right)=\left[l_{2}\right]$, the equivalence class of $L_{2} / \alpha_{1}\left(L_{1}\right)$ that contains $l_{2}$.

- $\delta$ is well defined; that is, $\delta\left(k_{3}\right)$ is independent of our choice of $m_{1} \in M_{1}$ and $l_{2} \in L_{2}$ satisfying the stated conditions.

Suppose that $g_{1}\left(m_{1}\right)=k_{3}=g_{1}\left(m_{1}^{\prime}\right)$ for some $m_{1}$ and $m_{1}^{\prime} \in M_{1}$, and that $f_{2}\left(l_{2}\right)=\alpha_{2}\left(m_{1}\right), f_{2}\left(l_{2}^{\prime}\right)=\alpha_{2}\left(m_{1}^{\prime}\right)$ for some $l_{2}, l_{2}^{\prime} \in L_{2}$. Since the second row is exact, there exist $l_{1}, l_{1}^{\prime} \in L_{1}$ such that $m_{1}+f_{1}\left(l_{1}\right)=m_{1}^{\prime}+f_{1}\left(l_{1}^{\prime}\right)$. We successively obtain the following equalities:

$$
\begin{aligned}
\alpha_{2}\left(m_{1}\right)+\left(\alpha_{2} \circ f_{1}\right)\left(l_{1}\right) & =\alpha_{2}\left(m_{1}^{\prime}\right)+\left(\alpha_{2} \circ f_{1}\right)\left(l_{1}^{\prime}\right), \\
f_{2}\left(l_{2}\right)+\left(f_{2} \circ \alpha_{1}\right)\left(l_{1}\right) & =f_{2}\left(l_{2}^{\prime}\right)+\left(f_{2} \circ \alpha_{1}\right)\left(l_{1}^{\prime}\right), \\
f_{2}\left(l_{2}+\alpha_{1}\left(l_{1}\right)\right) & =f_{2}\left(l_{2}^{\prime}+\alpha_{1}\left(l_{1}^{\prime}\right)\right), \quad\left(f_{2} \text { is a monomorphism }\right) \\
l_{2}+\alpha_{1}\left(l_{1}\right) & =l_{2}^{\prime}+\alpha_{1}\left(l_{1}^{\prime}\right), \\
{\left[l_{2}\right] } & =\left[l_{2}^{\prime}\right] .
\end{aligned}
$$

Thus $l_{2}$ and $l_{2}^{\prime}$ lie in the same equivalence class of $L_{2} / \alpha_{1}\left(L_{1}\right)$; that is, $\delta$ is well defined.

- We prove first that $\overline{g_{K}\left(\operatorname{Ker}\left(\alpha_{2}\right)\right)} \subseteq \operatorname{Ker}(\delta)$. Notice that it is enough to prove that $g_{K}\left(\operatorname{Ker}\left(\alpha_{2}\right)\right) \subseteq \operatorname{Ker}(\delta)$. Let $k_{3}=g_{K}\left(k_{2}\right)$ for some $k_{2} \in \operatorname{Ker}\left(\alpha_{2}\right)$. Since the definition of $\delta$ is independent of the choice of $m_{1} \in M_{1}$ and $l_{2} \in L_{2}$ satisfying the given conditions above and since $g_{1}\left(k_{2}\right)=g_{K}\left(k_{2}\right)=k_{3}$, we can choose $m_{1}=k_{2}$ and $l_{2}=0$. Notice that we have $f_{2}\left(l_{2}\right)=\alpha_{2}\left(m_{1}\right)=$ $\alpha_{2}\left(k_{2}\right)=0$, whence $l_{2}=0$ (recall that $\left.\operatorname{Ker}\left(f_{2}\right)=0\right)$. It follows that $\delta\left(k_{3}\right)=$ $\left[l_{2}\right]=[0]$. Consequently, $\overline{g_{K}\left(\operatorname{Ker}\left(\alpha_{2}\right)\right)} \subseteq \operatorname{Ker}(\delta)$. 
We prove now that $\operatorname{Ker}(\delta) \subseteq \overline{g_{K}\left(\operatorname{Ker}\left(\alpha_{2}\right)\right)}$. Let $k_{3} \in \operatorname{Ker}(\delta)$, and pick some $m_{1} \in M_{1}$ and $l_{2} \in L_{2}$ such that $g_{1}\left(m_{1}\right)=k_{3}$ and $f_{2}\left(l_{2}\right)=\alpha_{2}\left(m_{1}\right)$. Then, by assumption, $\left[l_{2}\right]=\delta\left(k_{3}\right)=[0]$; that is, $l_{2}+\alpha_{1}\left(l_{1}\right)=\alpha_{1}\left(l_{1}^{\prime}\right)$ for some $l_{1}, l_{1}^{\prime} \in$ $L_{1}$. We successively obtain the following equalities

$$
\begin{aligned}
f_{2}\left(l_{2}\right)+\left(f_{2} \circ \alpha_{1}\right)\left(l_{1}\right) & =\left(f_{2} \circ \alpha_{1}\right)\left(l_{1}^{\prime}\right), \\
\alpha_{2}\left(m_{1}\right)+\alpha_{2}\left(f_{1}\left(l_{1}\right)\right) & =\alpha_{2}\left(f_{1}\left(l_{1}^{\prime}\right)\right), \\
m_{1}+f_{1}\left(l_{1}\right)+k_{2} & =f_{1}\left(l_{1}^{\prime}\right)+k_{2}^{\prime}, \\
g_{1}\left(m_{1}\right)+g_{1}\left(k_{2}\right) & =g_{1}\left(k_{2}^{\prime}\right) \\
k_{3}+g_{K}\left(k_{2}\right) & =g_{K}\left(k_{2}^{\prime}\right) .
\end{aligned}
$$

Consequently, $\overline{g_{K}\left(\operatorname{Ker}\left(\alpha_{2}\right)\right)}=\operatorname{Ker}(\delta)$.

- Let $k_{3} \in \operatorname{Ker}\left(\alpha_{3}\right)$, and pick some $m_{1} \in M_{1}, l_{2} \in L_{2}$ such that $g_{1}\left(m_{1}\right)=k_{3}$ and $f_{2}\left(l_{2}\right)=\alpha_{2}\left(m_{1}\right)$. It follows that

$$
\left(f_{C} \circ \delta\right)\left(k_{3}\right)=f_{C}\left(\left[l_{2}\right]\right)=\left[f_{2}\left(l_{2}\right)\right]=\left[\alpha_{2}\left(m_{1}\right)\right]=[0] .
$$

Consequently, $\delta\left(\operatorname{Ker}\left(\alpha_{3}\right)\right) \subseteq \operatorname{Ker}\left(f_{C}\right)$. We claim that $\operatorname{Ker}\left(f_{C}\right) \subseteq \delta\left(\operatorname{Ker}\left(\alpha_{3}\right)\right)$. Let $\left[l_{2}\right] \in \operatorname{Ker}\left(f_{C}\right)$, so that $\left[f_{2}\left(l_{2}\right)\right]=f_{C}\left(\left[l_{2}\right]\right)=[0]$. By assumption, there $\underline{\text { exist } m_{1}}, m_{1}^{\prime} \in M_{1}$ such that $f_{2}\left(l_{2}\right)+\alpha_{2}\left(m_{1}\right)=\alpha_{2}\left(m_{1}^{\prime}\right)$. Since $\alpha_{2}\left(M_{1}\right)=$ $\overline{\alpha_{2}\left(M_{1}\right)}$, there exists $\widetilde{m}_{1} \in M_{1}$ such that $\alpha_{2}\left(\widetilde{m}_{1}\right)=f_{2}\left(l_{2}\right)$. It follows that $\left(\alpha_{3} \circ g_{1}\right)\left(\widetilde{m}_{1}\right)=\left(g_{2} \circ \alpha_{2}\right)\left(\widetilde{m}_{1}\right)=\left(g_{2} \circ f_{2}\right)\left(l_{2}\right)=0$. So $g_{1}\left(\widetilde{m}_{1}\right) \in \operatorname{Ker}\left(\alpha_{3}\right)$ and $\left[l_{2}\right]=\delta\left(g_{1}\left(\widetilde{m}_{1}\right)\right)$. Consequently, $\operatorname{Ker}\left(f_{C}\right)=\delta\left(\operatorname{Ker}\left(\alpha_{3}\right)\right)$.

- Suppose that $\delta\left(k_{3}\right)=\delta\left(k_{3}^{\prime}\right)$ for some $k_{3}, k_{3}^{\prime} \in \operatorname{Ker}\left(\alpha_{3}\right)$, and pick $m_{1}, m_{1}^{\prime} \in$ $M_{1}, l_{2}, l_{2}^{\prime} \in L_{2}$ such that $g_{1}\left(m_{1}\right)=k_{3}, g_{1}\left(m_{1}^{\prime}\right)=k_{3}^{\prime}, \alpha_{2}\left(m_{1}\right)=f_{2}\left(l_{2}\right)$, and $\alpha_{2}\left(m_{1}^{\prime}\right)=f_{2}\left(l_{2}^{\prime}\right)$. By assumption, $\left[l_{2}\right]=\left[l_{2}^{\prime}\right]$; that is, $l_{2}+\alpha_{1}\left(l_{1}\right)=l_{2}^{\prime}+\alpha_{1}\left(l_{1}^{\prime}\right)$ for some $l_{1}, l_{1}^{\prime} \in L_{1}$ and we successively obtain the following equalities:

$$
\begin{aligned}
f_{2}\left(l_{2}\right)+\left(f_{2} \circ \alpha_{1}\right)\left(l_{1}\right) & =f_{2}\left(l_{2}^{\prime}\right)+\left(f_{2} \circ \alpha_{1}\right)\left(l_{1}^{\prime}\right) & & \\
\alpha_{2}\left(m_{1}\right)+\left(\alpha_{2} \circ f_{1}\right)\left(l_{1}\right) & =\alpha_{2}\left(m_{1}^{\prime}\right)+\left(\alpha_{2} \circ f_{1}\right)\left(l_{1}^{\prime}\right) & & \\
m_{1}+f_{1}\left(l_{1}\right)+k_{2} & =m_{1}^{\prime}+f_{1}\left(l_{1}^{\prime}\right)+k_{2}^{\prime}, & & (\text { second column is exact }) \\
g_{1}\left(m_{1}\right)+g_{1}\left(k_{2}\right) & =g_{1}\left(m_{1}^{\prime}\right)+g_{1}\left(k_{2}^{\prime}\right), & & \left(g_{1} \circ f_{1}=0\right) \\
k_{3}+g_{K}\left(k_{2}\right) & =k_{3}^{\prime}+g_{K}\left(k_{2}^{\prime}\right) . & &
\end{aligned}
$$

The last statement in (4) follows since $g_{K}\left(\operatorname{Ker}\left(\alpha_{2}\right)\right) \subseteq \operatorname{Ker}(\delta)$.

(e) If $g_{K}\left(\operatorname{Ker}\left(\alpha_{2}\right)\right)=\overline{g_{K}\left(\operatorname{Ker}\left(\alpha_{2}\right)\right)}$, then $\operatorname{Ker}(\delta)=\overline{g_{K}\left(\operatorname{Ker}\left(\alpha_{2}\right)\right)}=g_{K}\left(\operatorname{Ker}\left(\alpha_{2}\right)\right)$. Suppose that $f_{C}\left[l_{2}\right]=f_{C}\left[l_{2}^{\prime}\right]$ for some $l_{2}, l_{2}^{\prime} \in L_{2}$. By definition, $\exists m_{1}, m_{1}^{\prime} \in M_{1}$ such that $f_{2}\left(l_{2}\right)+\alpha_{2}\left(m_{1}\right)=f_{2}\left(l_{2}^{\prime}\right)+\alpha_{2}\left(m_{1}^{\prime}\right)$. We successively obtain the following equalities:

$$
\begin{array}{rlrl}
\left(g_{2} \circ \alpha_{2}\right)\left(m_{1}\right) & =\left(g_{2} \circ \alpha_{2}\right)\left(m_{1}^{\prime}\right) & & \left(g_{2} \circ f_{2}=0\right) \\
\left(\alpha_{3} \circ g_{1}\right)\left(m_{1}\right) & =\left(\alpha_{3} \circ g_{1}\right)\left(m_{1}^{\prime}\right), & & \\
g_{1}\left(m_{1}\right)+k_{3} & =g_{1}\left(m_{1}^{\prime}\right)+k_{3}^{\prime}, & \\
g_{1}\left(m_{1}+\widetilde{m}_{1}\right) & =g_{1}\left(m_{1}^{\prime}+\widehat{m}_{1}\right) & & \\
m_{1}+\widetilde{m}_{1}+f_{1}\left(\widetilde{l}_{1}\right) & =m_{1}^{\prime}+\widehat{m}_{1}+f_{1}\left(\widehat{l}_{1}\right), & \\
\alpha_{2}\left(m_{1}\right)+\alpha_{2}\left(\widetilde{m}_{1}\right)+\left(\alpha_{2} \circ f_{1}\right)\left(\widetilde{l}_{1}\right) & =\alpha_{2}\left(m_{1}^{\prime}\right)+\alpha_{2}\left(\widehat{m}_{1}\right)+\left(\alpha_{2} \circ f_{1}\right)\left(\widehat{l}_{1}\right),
\end{array}
$$




$$
\begin{aligned}
f_{2}\left(l_{2}^{\prime}\right)+\alpha_{2}\left(m_{1}\right)+\alpha_{2}\left(\widetilde{m}_{1}\right)+\left(f_{2} \circ \alpha_{1}\right)\left(\widetilde{l}_{1}\right) & =\left[f_{2}\left(l_{2}^{\prime}\right)+\alpha_{2}\left(m_{1}^{\prime}\right)\right]+\alpha_{2}\left(\widehat{m}_{1}\right) \\
& +\left(f_{2} \circ \alpha_{1}\right)\left(\widehat{l}_{1}\right), \\
f_{2}\left(l_{2}^{\prime}\right)+\alpha_{2}\left(m_{1}\right)+\alpha_{2}\left(\widetilde{m}_{1}\right)+\left(f_{2} \circ \alpha_{1}\right)\left(\widetilde{l}_{1}\right) & =f_{2}\left(l_{2}\right)+\alpha_{2}\left(m_{1}\right)+\alpha_{2}\left(\widehat{m}_{1}\right) \\
& +\left(f_{2} \circ \alpha_{1}\right)\left(\widehat{l}_{1}\right), \\
f_{2}\left(l_{2}^{\prime}\right)+\alpha_{2}\left(\widetilde{m}_{1}\right)+\left(f_{2} \circ \alpha_{1}\right)\left(\widetilde{l}_{1}\right) & =f_{2}\left(l_{2}\right)+\alpha_{2}\left(\widehat{m}_{1}\right)+\left(f_{2} \circ \alpha_{1}\right)\left(\widehat{l}_{1}\right), \\
f_{2}\left(l_{2}^{\prime}+\widetilde{l}_{2}+\alpha_{1}\left(\widetilde{l}_{1}\right)\right)= & f_{2}\left(l_{2}+\widehat{l}_{2}+\alpha_{1}\left(\widetilde{l}_{1}\right)\right), \\
l_{2}^{\prime}+\widetilde{l}_{2}+\alpha_{1}\left(\widetilde{l}_{1}\right)= & l_{2}+\widehat{l}_{2}+\alpha_{1}\left(\widehat{l}_{1}\right) \quad\left(f_{2} \text { is injective }\right) \\
{\left[l_{2}^{\prime}\right]+\left[\widetilde{l}_{2}\right]=} & {\left[l_{2}\right]+\left[\widehat{l}_{2}\right], } \\
{\left[l_{2}^{\prime}\right]+\delta\left(k_{3}\right) } & =\left[l_{2}\right]+\delta\left(k_{3}^{\prime}\right) .
\end{aligned}
$$

The result follows since $\delta\left(\operatorname{Ker}\left(\alpha_{3}\right)\right) \subseteq \operatorname{Ker}\left(f_{C}\right)$.

\section{Additional Remarks}

4.1. Our homological lemmas are new, taking into consideration the well-known fact that $S$-SMod is, in general, not exact in the sense of [16], not semi-abelian in the sense of [11], and not homological in the sense of [4]. Moreover, they cannot be obtained via results on relative homological categories in the sense of $[\mathbf{9}]$; in particular, this applies to the results of $[\mathbf{1 0}]$.

4.2. Our homological lemmas allow investigating new notions for semimodules over semirings (e.g., normally flatness [1] ). This was in fact one of the main motivations behind this paper.

4.3. S-SMod is Barr-exact [3] ([8]) with canonical factorization system (Surj, Inj), where Surj is the class of surjective morphisms (regular epimorphisms) and Inj is the class of injective morphisms (monomorphisms). Moreover, S-SMod is homological in the sense of [7].

4.4. Our definition of exact sequences in $S$-SMod is based on analyzing the notion of an exact sequence in an arbitrary pointed category relative to a given factorization system. It is consistent with the notion of an exact sequence in an arbitrary pointed regular Barr-exact category with finite limits $[4,4.1 .7]$. Moreover, our notion of a short exact sequence $0 \longrightarrow A \longrightarrow B \longrightarrow C \longrightarrow 0$ coincides with that of an extension in the sense of $[\mathbf{1 9}]$ (see also $[\mathbf{1 3}]$ ).

4.5. Being a Barr-exact category, a natural tool to study exactness in $S$-SMod is that of an exact fork [3]. However, since $S$-SMod has additional features, one still expects to deal with exact sequences rather than the more complicated exact forks.

\section{Acknowledgments}

The author appreciates the efforts of the editor/referee for the feedback which significantly improved the revised version of the paper. Moreover, he thanks all the mathematicians who clarified to him some issues related to the nature of the categories of semimodules and exact sequences, especially G. Janelidze, F. Linton, A. Patchkoria, H. Porst, and R. Wisbauer. 


\section{References}

[1] J. Abuhlail, Some Remarks on Tensor Products and Flatness of Semimodules, Semigroup Forum, in Press.

[2] J. Adámek, H. Herrlich and G. E. Strecker, Abstract and Concrete Categories, Dover Publications Edition, 2009.

[3] M. Barr, Exact categories, in Exact Categories and Categories of Sheaves, Lec. Not. Math. 236, Springer (1971), 1-120.

[4] F. Borceux and D. Bourn, Mal'cev, Protomodular, Homological and SemiAbelian Categories, Mathematics and its Application 566, Kluwer Academic Publishing, 2004.

[5] B. Csákány (B. Čakan'), Primitive classes of algebras which are equivalent to classes of semi-modules and modules (in Russian), Acta Sci. Math. (Szeged) 24 (1963), 157-164.

[6] J. Golan, Semirings and their Applications, Kluwer Academic Publishers, 1999.

[7] M. Grandis, Homological Algebra: In Strongly Non-Abelian Settings, World Scientific Publishing Company, 2013.

[8] P.A. Grillet, Regular categories, in Exact Categories and Categories of Sheaves, Lec. Not. Math. 236, Springer (1971), 121-222.

[9] T. Janelidze, Relative homological categories, J. Homotopy Relat. Struct. 1(1) (2006), 185-194.

[10] T. Janelidze, Snake lemma in incomplete relative homological categories, Theory Appl. Categ. 23(4) (2010), 76-91.

[11] G. Janelidze, L. Márki and W. Tholen, Semi-abelian categories, J. Pure Appl. Algebra 168(2-3) (2002), 367-386.

[12] S. Mac Lane, Categories for the Working Mathematician, 2nd edition, Graduate Texts in Mathematics 5, Springer-Verlag, 1998.

[13] A. Patchkoria, Extensions of semimodules and the Takahashi functor $\operatorname{Ext}_{\Lambda}(C, A)$, Homology Homotopy Appl. 5(1) (2003), 387-406.

[14] A. Patchkoria, On exactness of long sequences of homology semimodules, J. Homotopy Relat. Struct. 1(1) (2006), 229-243.

[15] K. B. Patil and R. P. Deore, Some results on semirings and semimodules, Bull. Calcutta Math. Soc. 98(1) (2006), 49-56.

[16] D. Puppe, Korrespondenzen in abelschen Kategorien, Math. Ann. 148 (1962), $1-30$.

[17] H. Schubert, Categories, Springer Verlag, 1972.

[18] M. Takahashi, On the bordism categories, II, Elementary properties of semimodules, Math. Sem. Notes Kobe Univ. 9(2) (1981), 495-530.

[19] M. Takahashi, Extensions of semimodules. I, Math. Sem. Notes Kobe Univ. 10(2) (1982), 563-592. 
Jawad Y. Abuhlail abuhlail@kfupm.edu.sa

Department of Mathematics and Statistics, King Fahd University of Petroleum \& Minerals, Box \#5046, KFUPM, 31261 Dhahran, KSA 